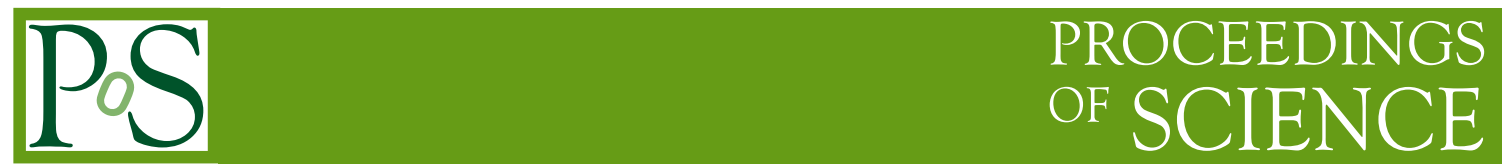

\title{
Charged Higgs production: higher-order corrections
}

\section{Nikolaos Kidonakis*}

Kennesaw State University, Physics \#1202, Kennesaw, GA 30144-5591,USA

E-mail: nkidonak@kennesaw.edu

\begin{abstract}
I present a calculation of higher-order radiative corrections to charged Higgs production in association with a top quark via the process $b g \rightarrow t H^{-}$. Results for charged Higgs production at the LHC are presented, including the dependence of the cross section on the charged Higgs mass, the top quark mass, the factorization and renormalization scales, and $\tan \beta$. I show that the theoretical prediction for the cross section is significantly enhanced and is greatly stabilized when the higher-order corrections are included.
\end{abstract}

International Europhysics Conference on High Energy Physics

July 21st - 27th 2005

Lisboa, Portugal

${ }^{*}$ Speaker. 


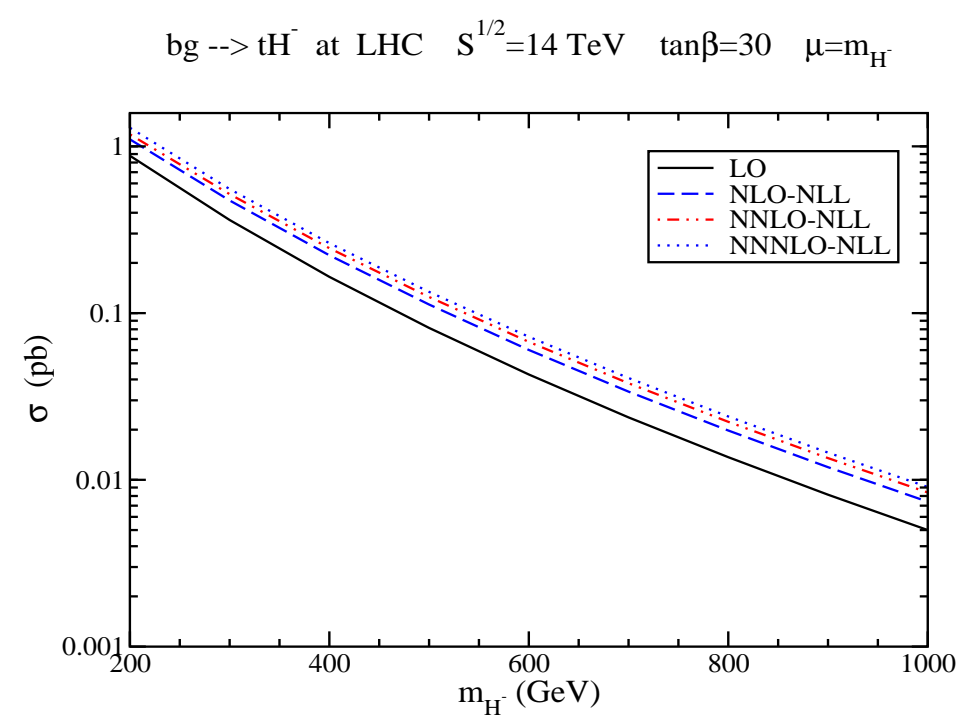

Figure 1: The cross section for charged Higgs production via $b g \rightarrow t H^{-}$at the LHC.

\section{Charged Higgs production via $b g \rightarrow t H^{-}$}

A future observation of a charged Higgs boson at the LHC would be a clear sign of new physics beyond the Standard Model [1]. The LHC has a good potential for discovery of a charged Higgs boson in association with a top quark via bottom-gluon fusion, $b g \rightarrow t H^{-}$.

The lowest-order cross section is proportional to $\alpha \alpha_{s}\left(m_{b}^{2} \tan ^{2} \beta+m_{t}^{2} \cot ^{2} \beta\right)$ where $m_{b}$ is the bottom quark mass, $m_{t}$ is the top quark mass, and $\tan \beta=v_{2} / v_{1}$ is the ratio of the vacuum expectation values, $v_{2}, v_{1}$, for the two Higgs doublets in the MSSM. SUSY and QCD corrections to this process were calculated in [2].

Threshold corrections $[3,4]$ are expected to make significant contributions to cross sections of processes with very massive final states; known examples include top quark [5] and $W$-boson [6] production, among others. Here we calculate higher-order soft-gluon threshold corrections to the charged Higgs cross section through next-to-next-to-next-to-leading order (NNNLO) [7, 8].

For the process $b\left(p_{b}\right)+g\left(p_{g}\right) \longrightarrow t\left(p_{t}\right)+H^{-}\left(p_{H^{-}}\right)$we define $s=\left(p_{b}+p_{g}\right)^{2}, t=\left(p_{b}-p_{t}\right)^{2}$, $u=\left(p_{g}-p_{t}\right)^{2}$ and $s_{4}=s+t+u-m_{t}^{2}-m_{H^{-}}{ }^{2}$. We use the $\overline{\mathrm{MS}}$ bottom quark mass $m_{b}$ in the coupling but set $m_{b}=0$ in the kinematics. At threshold $s_{4} \rightarrow 0$. The soft corrections are logarithmic terms of the form $\left[\ln ^{l}\left(s_{4} / m_{H^{-}}{ }^{2}\right) / s_{4}\right]+$ where $l \leq 2 n-1$ for the order $\alpha_{s}^{n}$ corrections. The leading logarithms (LL) are those with $l=2 n-1$ while the next-to-leading logarithms (NLL) are those with $l=2 n-2$.

\section{Cross section for $b g \rightarrow t H^{-}$at the $\mathbf{L H C}$}

Now we calculate the cross section for $b g \rightarrow t H^{-}$at the LHC including NLO, NNLO, and NNNLO soft-gluon corrections at NLL accuracy. We use the MRST2002 approximate NNLO 


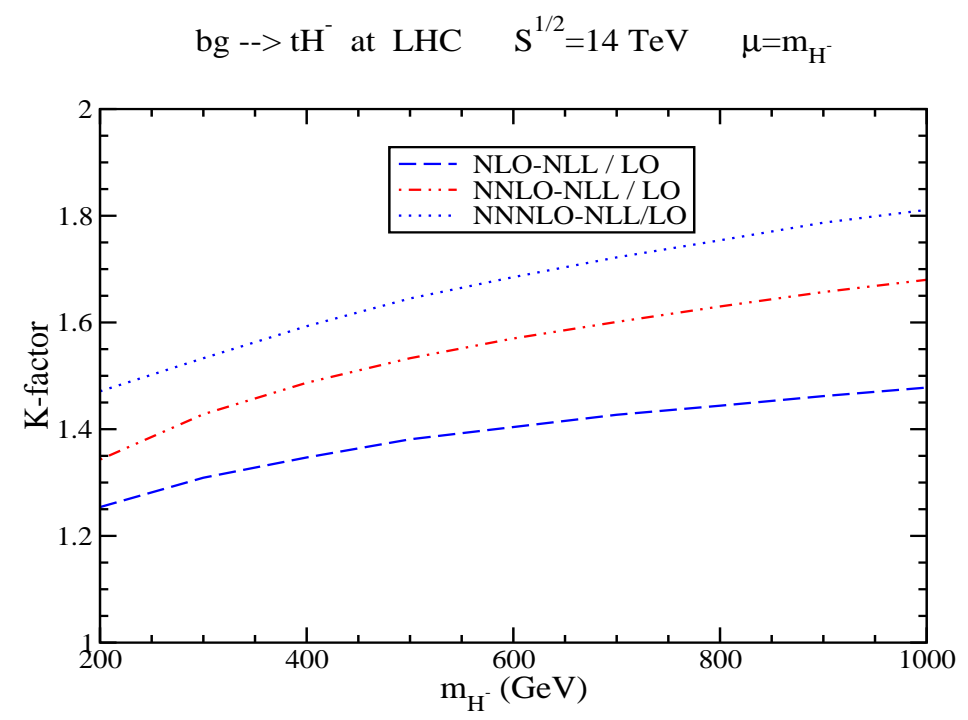

Figure 2: The $K$-factors for charged Higgs production via $b g \rightarrow t H^{-}$at the LHC.

parton distributions functions [9] with the respective three-loop evaluation of $\alpha_{s}$.

In Figure 1 we plot the cross section versus charged Higgs mass for $p p$ collisions at the LHC with $\sqrt{S}=14 \mathrm{TeV}$. We set the factorization scale equal to the renormalization scale and denote this common scale by $\mu$. We show results for the LO, NLO-NLL, NNLO-NLL, and NNNLO-NLL cross sections, all with a choice of scale $\mu=m_{H^{-}}$. We use the same NNLO parton densities and couplings in all the results, so that we can concentrate on the effects of the soft-gluon corrections. In the calculations we choose a value $\tan \beta=30$. It is straightforward to calculate the cross section for any other value of $\tan \beta$, since the only dependence on $\beta$ is in a factor $m_{b}^{2} \tan ^{2} \beta+m_{t}^{2} \cot ^{2} \beta$ appearing in the LO term. The NLO, NNLO, and NNNLO threshold corrections are positive and provide a significant enhancement to the lowest-order result. The cross sections for the related process $\bar{b} g \rightarrow \bar{t} H^{+}$are exactly the same.

The relative size of the corrections is better shown in Figure 2 where we plot the $K$-factors, i.e. ratios of cross sections at various orders. As expected the corrections increase for higher charged Higgs masses since then we get closer to threshold. The NNNLO-NLL / LO curve shows that if we include all NLL threshold corrections through NNNLO we get an enhancement over the lowest-order result of approximately $45 \%$ to $80 \%$, depending on the charged Higgs mass.

In Figure 3 the scale dependence of the charged Higgs cross section is shown. We see that the scale dependence diminishes progressively as we move from LO to NNNLO and while it is rather large at LO, at NNNLO it is insignificant.

Finally, we note that the cross section decreases with increasing top quark mass, but this dependence is not very strong. 


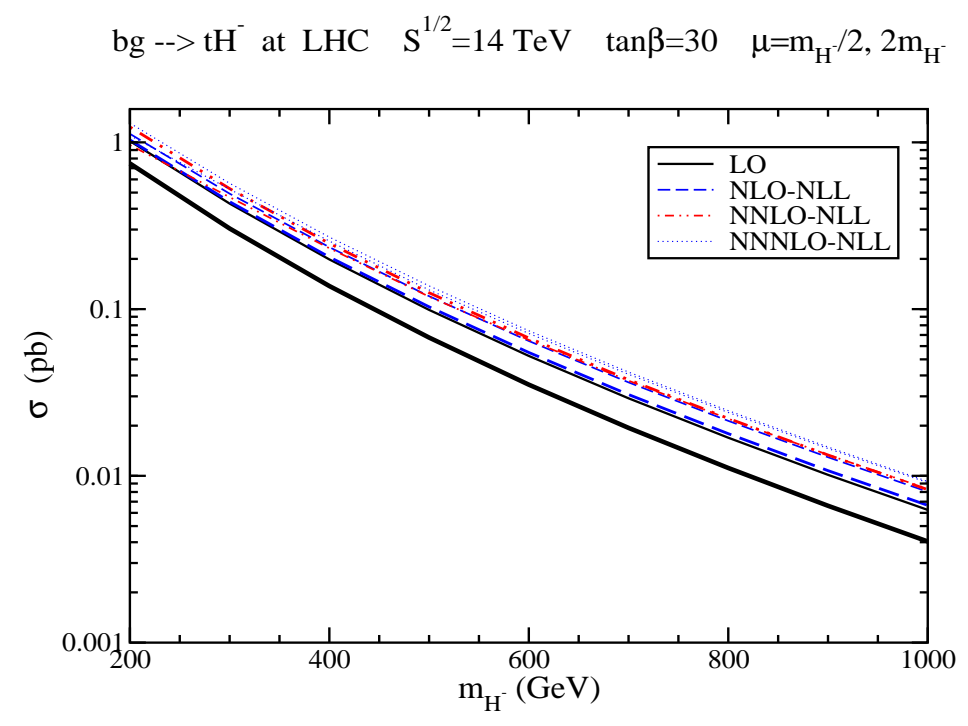

Figure 3: Scale dependence of charged Higgs production via $b g \rightarrow t H^{-}$at the LHC.

\section{References}

[1] The Higgs Working Group, in Les Houches 2003, hep-ph/0406152.

[2] A. Belyaev, D. Garcia, J. Guasch, and J. Sola, Phys. Rev. D 65, 031701 (2002) [hep-ph/0105053]; JHEP 06, 059 (2002) [hep-ph/0203031]; S. Zhu, Phys. Rev. D 67, 075006 (2003) [hep-ph/0112109]; T. Plehn, Phys. Rev. D 67, 014018 (2003) [hep-ph/0206121]; E.L. Berger, T. Han, J. Jiang, and T. Plehn, Phys. Rev. D 71, 115012 (2005) [hep-ph/0312286].

[3] N. Kidonakis and G. Sterman, Phys. Lett. B 387, 867 (1996); Nucl. Phys. B 505, 321 (1997) [hep-ph/9705234]; N. Kidonakis, Int. J. Mod. Phys. A 151245 (2000)[hep-ph/9902484].

[4] E. Laenen, G. Oderda, and G. Sterman, Phys. Lett. B 438, 173 (1998) [hep-ph/9806467].

[5] N. Kidonakis, Phys. Rev. D 64, 014009 (2001) [hep-ph/0010002]; in DPF 2000, Int. J. Mod. Phys. A 16, s1A, 363 (2001) [hep-ph/0009013]; N. Kidonakis and R. Vogt, Phys. Rev. D 68, 114014 (2003) [hep-ph/0308222]; in DPF 2004, Int. J. Mod. Phys. A 20, 3171 (2005) [hep-ph/0410367].

[6] N. Kidonakis and A. Sabio Vera, JHEP 02, 027 (2004) [hep-ph/0311266]; R.J. Gonsalves, N. Kidonakis, and A. Sabio Vera, Phys. Rev. Lett. (in press) [hep-ph/0507317].

[7] N. Kidonakis, Int. J. Mod. Phys. A 19, 1793 (2004) [hep-ph/0303186]; Mod. Phys. Lett. A 19, 405 (2004) [hep-ph/0401147]; in DIS 2005, hep-ph/0506299; hep-ph/0509079.

[8] N. Kidonakis, JHEP 05, 011 (2005) [hep-ph/0412422]; in DIS 2004 [hep-ph/0406179]; in DIS 2005, hep-ph/0505271.

[9] A.D. Martin, R.G. Roberts, W.J. Stirling, and R.S. Thorne, Eur. Phys. J. C 28, 455 (2003) [hep-ph/0211080]. 